\title{
ENTIRE FUNCTIONS AND THEIR FIRST DERIVATIVES SHARING SIMPLE $\beta$-POINTS FOR A SMALL FUNCTION $\beta$
}

\author{
Amer H. H. Al-Khaladi
}

\begin{abstract}
The following theorem has been proved by A. Schweizer [7]. If a nonconstant entire function $f$ and its derivative $f^{\prime}$ share their simple zeros and if every simple $a$ point of $f$ is a (not necessarily simple) $a$-point of $f^{\prime}$ for some nonzero constant $a$, then $f \equiv f^{\prime}$. In this paper we shall prove that the above result is also true when the nonzero constant $a$ is replaced by a meromorphic small function $\beta(\not \equiv 0, \infty)$.
\end{abstract}

\section{Introduction and results}

In this paper, we use the same notation as given in Nevanlinna theory of meromorphic functions ([2, 8]). For a meromorphic function $h$, let $T(r, h)$ denote the Nevanlinna characteristic of $h$ and let $S(r, h)$ be any quantity satisfying $S(r, h)=o\{T(r, h)\}$ as $r \rightarrow \infty$, except possibly on a set of finite linear measure. Then a meromorphic function $\beta$ is called a small function of $h$ if and only if $T(r, h)=S(r, h)$. Let $f$ and $g$ be two nonconstant entire functions. For $a \in \mathbf{C}$ we say that $f$ and $g$ share the value $a \mathrm{CM}$ (counting multiplicities) if the $a$ points of $f$ and $g$ coincide in locations and multiplicities. If we do not consider the multiplicities, we say that $f$ and $g$ share the value $a$ IM (ignoring multiplicities). Let $k$ be a positive integer and always keeping in mind that the $\beta$ points of $f$ are the zeros of $f-\beta$ and their multiplicity is the multiplicity of that zero. We denote by $N_{k)}\left(r, \frac{1}{f-\beta}\right)$ the counting function of $\beta$-points of $f$ with multiplicity $\leq k$, by $N_{(k+1}\left(r, \frac{1}{f-\beta}\right)$ the counting function of $\beta$-points of $f$ with multiplicity $>k$ and by $N_{=k}\left(r, \frac{1}{f-\beta}\right)$ counting function of $\beta$-points of $f$ which have the multiplicity $k$. In the same way we define $\bar{N}_{k)}\left(r, \frac{1}{f-\beta}\right)$,

2010 Mathematics Subject Classification. 30D35.

Key words and phrases. Entire function; simple sharing; small function; uniqueness theorem. Received July 11, 2014; revised October 7, 2014. 
$\bar{N}_{(k+1}\left(r, \frac{1}{f-\beta}\right)$ and $\bar{N}_{=k}\left(r, \frac{1}{f-\beta}\right)$ where in counting the $\beta$-points of $f$ we ignore the multiplicities ([8]). Finally we denote by $E(\beta, f)$ the set of $\beta$-points of $f$ (counting multiplicities), by $\bar{E}(\beta, f)$ the set of $\beta$-points of $f$ (ignoring multiplicities) and by $E_{1)}(\beta, f)$ the set of $\beta$-points of $f$ with multiplicity one. Thus we say that $f$ and $g$ share $\beta \mathrm{CM}, \beta \mathrm{IM}, \beta$ simple if $E(\beta, f)=E(\beta, g)$, $\bar{E}(\beta, f)=\bar{E}(\beta, g)$ and $E_{1)}(\beta, f)=E_{1)}(\beta, g)$ respectively.

On the problems of uniqueness of an entire function and its derivative that share some values. Rubel-Yang ([6]) proved that if the entire function $f$ and $f^{\prime}$ share two distinct finite values CM then $f \equiv f^{\prime}$. Mues-Steinmetz ([3]) improved this result to the case when $f$ and $f^{\prime}$ share two distinct finite values IM. ZhengWang ([9]) generalized this result to $f$ and $f^{\prime}$ which share two small functions CM. In 2000 Qiu ([5]) improved this result to the case when $f$ and $f^{\prime}$ share two small functions IM. Recently, Schweizer [7] proved the following theorem:

THEOREM 1.1. Let $f$ be a nonconstant entire function and let a be a nonzero constant. If $f$ and $f^{\prime}$ share their simple zeros and if every simple a-point of $f$ is a (not necessarily simple) a-point of $f^{\prime}$, then $f \equiv f^{\prime}$.

It is natural to ask whether the "nonzero constant $a$ " of Theorem 1.1 can be replaced by "small function $\beta(\not \equiv 0, \infty)$ "? In this paper, we will give a positive answer to this question. Indeed, we shall prove the following:

THEOREM 1.2. Let $f$ be a nonconstant entire function and let $\beta$ be a small meromorphic function of $f$ such that $\beta(z) \not \equiv 0, \infty$. If $f$ and $f^{\prime}$ share their simple zeros and if every simple $\beta$-point of $f$ is a (not necessarily simple) $\beta$-point of $f^{\prime}$, then $f \equiv f^{\prime}$.

It is obvious that Theorem 1.2 is a generalization of Theorem 1.1. From Theorem 1.2, we directly conclude the following corollary:

COROLlary 1.3. Let $f$ be a nonconstant entire function and let $\beta$ be a small meromorphic function of $f$ such that $\beta(z) \not \equiv 0, \infty$. If $E_{1)}(0, f)=E_{1)}\left(0, f^{\prime}\right)$ and $E_{1)}(\beta, f)=E_{1)}\left(\beta, f^{\prime}\right)$, then $f \equiv f^{\prime}$.

A. Schweizer [7] also gave the following example:

Example 1. Let

$$
f(z)=\frac{a}{2}(\sin (2 z)+1), \quad 0 \neq a \in \mathbf{C}
$$

then $f^{\prime}(z)=a \cos (2 z)$. All $a$-points of $f$ and of $f^{\prime}$ and all zeros of $f$ have multiplicity 2. Thus the condition that $f$ and $f^{\prime}$ share their simple $a$-points and that if every simple zero of $f$ is a simple zero of $f^{\prime}$ does not imply $f \equiv f^{\prime}$. 
Note that from (1.1) we see that

$$
L=\frac{\left(\frac{f-a}{0-a}\right)^{\prime}}{\frac{f-a}{0-a}}=\frac{f^{\prime}}{f-a}=\frac{2 \cos (2 z)}{\sin (2 z)-1} .
$$

Hence $L^{\prime}=\frac{4}{\sin (2 z)-1}$, and so $\bar{N}\left(r, \frac{1}{L^{\prime}}\right)=S(r, f)$. In the present paper, we shall prove the following theorem which includes Example 1 as a special case:

THEOREM 1.4. Let $f$ be a nonconstant entire function and let $\beta_{1}$ and $\beta_{2}$ be two distinct small meromorphic functions of $f$. If all zeros of $f-\beta_{1}$ and $f-\beta_{2}$ have multiplicities greater than 1, then either

$$
f(z)-\beta_{1}(z)=\frac{-1}{4 A}\left(\beta_{2}(z)-\beta_{1}(z)\right) e^{-c z}\left(1-A e^{c z}\right)^{2}
$$

and

$$
f(z)-\beta_{2}(z)=\frac{-1}{4 A}\left(\beta_{2}(z)-\beta_{1}(z)\right) e^{-c z}\left(1+A e^{c z}\right)^{2}
$$

or

$$
T(r, f) \leq 4 \bar{N}\left(r, \frac{1}{L^{\prime}}\right)+S(r, f)
$$

where $A, c$ are nonzero constants, $L=\frac{F^{\prime}}{F}$ and $F=\frac{f-\beta_{1}}{\beta_{2}-\beta_{1}}$.

From Theorem 1.4, we immediately deduce the following corollary:

COROLlary 1.5. Let $f$ be a nonconstant entire function, and let $\beta_{1}$ and $\beta_{2}$ be two distinct small meromorphic functions of $f$ satisfying $\bar{N}\left(r, \frac{1}{L^{\prime}}\right)=S(r, f)$ where $L=\frac{F^{\prime}}{F}$ and $F=\frac{f-\beta_{1}}{\beta_{2}-\beta_{1}}$. If all zeros of $f-\beta_{1}$ and $f-\beta_{2}$ have multiplicities greater than 1, then (1.2) and (1.3) hold.

Remarks.

1. If $\beta_{1} \equiv a, \beta_{2} \equiv 0, c=2 i$ and $A=-i$, then (1.3) becomes (1.1).

2. From (1.2) or (1.3) it is easy to see that $L^{\prime}(z)=\frac{-2 A c^{2} e^{c z}}{\left(1-A e^{c z}\right)^{2}}$. Hence $\bar{N}\left(r, \frac{1}{L^{\prime}}\right)=S(r, f)$. 

3. The following example shows that the condition $\bar{N}\left(r, \frac{1}{L^{\prime}}\right)=S(r, f)$ in
Corollary 1.5 cannot be removed.

Example 2. Let $\alpha(z)$ be any nonconstant entire function, $A$ be any nonzero constant and

$$
f(z)-\beta_{1}(z)=\frac{-1}{4 A}\left(\beta_{2}(z)-\beta_{1}(z)\right) e^{-\int_{0}^{z} \alpha(t) d t}\left(1-A e^{\int_{0}^{z} \alpha(t) d t}\right)^{2} .
$$

Then it is easy to see that all zeros of $f-\beta_{1}$ and $f-\beta_{2}$ have multiplicities 2 and

$$
L(z)=-2 A \alpha\left(\frac{1}{2 A}+\frac{e^{\int_{0}^{z} \alpha(t) d t}}{1-A e^{\int_{0}^{z} \alpha(t) d t}}\right) .
$$

Hence

$$
L^{\prime}(z)=\frac{-\alpha^{\prime}\left[1+\frac{\alpha^{4}}{\alpha^{\prime 2}}-\left(A e^{\int_{0}^{z} \alpha(t) d t}-\frac{\alpha^{2}}{\alpha^{\prime}}\right)^{2}\right]}{\left(1-A e^{\int_{0}^{z} \alpha(t) d t}\right)^{2}} .
$$
From this we deduce that $\bar{N}\left(r, \frac{1}{L^{\prime}}\right) \neq S(r, f)$ but the conclusion of Corollary 1.5
is not valid.

\section{Some lemmas}

For the proof of our theorems we need the following lemmas:

LeMma 2.1 [4]. Let $f$ be a nonconstant meromorphic function and $\Psi=$ $a_{n} f^{n}+a_{n-1} f^{n-1}+\cdots+a_{1} f+a_{0}$, where $a_{n} \neq \equiv 0, a_{n-1}, \ldots, a_{1}, a_{0}$ are meromorphic small functions of $f$. If $\bar{N}\left(r, \frac{1}{\Psi}\right)=S(r, f)$, then three cases are possible

(i) $\Psi=a_{n}\left(f+\frac{a_{n-1}}{n a_{n}}\right)^{n}$;

(ii) There exist a meromorphic small function $\alpha_{0} \not \equiv 0$ and an integer $\mu$ such that $n=2 \mu$ and

$$
\Psi=a_{n}\left(f^{2}+2 \frac{a_{n-1}}{n a_{n}} f+\left(\frac{a_{n-1}}{n a_{n}}\right)^{2}+\alpha_{0}\right)^{\mu}
$$

(iii) There exist a meromorphic small function $\alpha_{0} \not \equiv 0$, positive integers $\mu_{1}$ and $\mu_{2}$, and distinct complex numbers $\lambda_{1}$ and $\lambda_{2}$ such that $\mu_{1}+\mu_{2}=n, \mu_{1} \lambda_{1}+\mu_{2} \lambda_{2}=0$, and

$$
\Psi=a_{n}\left(f+\frac{a_{n-1}}{n a_{n}}-\lambda_{1} \alpha_{0}\right)^{\mu_{1}}\left(f+\frac{a_{n-1}}{n a_{n}}-\lambda_{2} \alpha_{0}\right)^{\mu_{2}} .
$$


ENTIRE FUNCTIONS AND THEIR FIRST DERIVATIVES SHARING SIMPLE $\beta$-POINTS 293

Lemma 2.2 [2, P. 47]. Let $f$ be a nonconstant meromorphic function, and $a_{1}$, $a_{2}, a_{3}$ be distinct small functions of $f$. Then

$$
T(r, f) \leq \sum_{j=1}^{3} \bar{N}\left(\frac{1}{f-a_{j}}\right)+S(r, f) .
$$

LEMma 2.3 [5]. Let $f$ be a nonconstant entire function, $\alpha_{1}$ and $\alpha_{2}$ be two distinct small functions of $f$ with $\alpha_{1} \not \equiv \infty$ and $\alpha_{2} \not \equiv \infty$. Set

$$
\Delta(f)=\left|\begin{array}{ll}
f-\alpha_{1} & \alpha_{1}-\alpha_{2} \\
f^{\prime}-\alpha_{1}^{\prime} & \alpha_{1}^{\prime}-\alpha_{2}^{\prime}
\end{array}\right|=\left|\begin{array}{ll}
f-\alpha_{2} & \alpha_{1}-\alpha_{2} \\
f^{\prime}-\alpha_{2}^{\prime} & \alpha_{1}^{\prime}-\alpha_{2}^{\prime}
\end{array}\right|
$$

Then

$$
\Delta(f) \not \equiv 0 \quad \text { and } \quad m\left(r, \frac{\Delta(f)}{f-\alpha_{i}}\right)=S(r, f) \quad(i=1,2)
$$

Lemma $2.4[1]$. Let $f^{\prime}$ be a nonconstant meromorphic function and let

$$
\Gamma=\left(\frac{f^{\prime \prime}}{f^{\prime}}\right)^{2}-2\left(\frac{f^{\prime \prime}}{f^{\prime}}\right)^{\prime}
$$

Then

$$
T(r, \Gamma) \leq 2 \bar{N}\left(r, \frac{1}{f^{\prime}}\right)+2 \bar{N}_{(2}(r, f)+S\left(r, f^{\prime}\right)
$$

\section{Proof of Theorems}

\subsection{Proof of Theorem 1.2}

The proof here is by contradiction. Assume that $f \not \equiv f^{\prime}$. Set

$$
W=\frac{\left[(f / \beta)^{\prime}\right]^{2}\left(f-f^{\prime}\right)}{f^{2}(f-\beta)} .
$$

From Nevanlinna's fundamental estimate of the logarithmic derivative we obtain

$$
\begin{aligned}
m(r, W) & \leq m\left(r, \frac{1}{\beta} \cdot \frac{(f / \beta)^{\prime}}{(f / \beta)}\right)+m\left(r, \frac{1}{\beta} \cdot \frac{(f / \beta)^{\prime}}{((f / \beta)-1)}\right)+m\left(r, 1-\frac{f^{\prime}}{f}\right)+O(1) \\
& \leq 2 m\left(r, \frac{1}{\beta}\right)+S(r, f)=S(r, f)
\end{aligned}
$$

Since $f$ and $f^{\prime}$ share their simple zeros, every zero of $f$ has multiplicity at least 3 and $f^{\prime}$ has no simple zeros. That is

$$
N\left(r, \frac{1}{f}\right)=N_{(3}\left(r, \frac{1}{f}\right) \quad \text { and } \quad N\left(r, \frac{1}{f^{\prime}}\right)=N_{(2}\left(r, \frac{1}{f^{\prime}}\right) .
$$


Further it follows from (3.1) that if $z_{0}$ is a zero of $f$ with multiplicity $p \geq 3$ and $\beta\left(z_{0}\right) \neq 0, \infty$, then

$$
W(z)=O\left(\left(z-z_{0}\right)^{p-3}\right), \quad \text { as } z \rightarrow z_{0} .
$$

Since every simple zero of $f-\beta$ is a zero of $f^{\prime}-\beta$, we can also conclude from (3.1) that if $z_{1}$ is a zero of $f-\beta$ with multiplicity $q \geq 1$ and $\beta\left(z_{1}\right) \neq 0, \infty$,

$$
W(z)= \begin{cases}O(1) & \text { if } q=1 \\ O\left(\left(z-z_{1}\right)^{q-2}\right) & \text { if } q \geq 2\end{cases}
$$

as $z \rightarrow z_{1}$. Thus, from (3.2), (3.4) and (3.5) we get

$$
\begin{aligned}
T(r, W) & =N(r, W)+m(r, W)=N(r, W)+S(r, f) \\
& \leq 5 N\left(r, \frac{1}{\beta}\right)+S(r, f) \\
& \leq 5 T(r, \beta)+S(r, f)=S(r, f) .
\end{aligned}
$$

We can rewrite (3.1) in the form

$$
W\left(\frac{\beta-f}{f}\right)=\left(\frac{(f / \beta)^{\prime}}{f}\right)^{2}\left(\frac{f^{\prime}}{f}-1\right)=\frac{1}{\beta^{2}}\left(\frac{f^{\prime}}{f}-\frac{\beta^{\prime}}{\beta}\right)^{2}\left(\frac{f^{\prime}}{f}-1\right),
$$

or

$$
\begin{aligned}
G & =(g-1)(g-\lambda)^{2}+W \beta^{2} \\
& =g^{3}-(2 \lambda+1) g^{2}+\lambda(\lambda+2) g+W \beta^{2}-\lambda^{2},
\end{aligned}
$$

where $G=\frac{W \beta^{3}}{f}, g=\frac{f^{\prime}}{f}$ and $\lambda=\frac{\beta^{\prime}}{\beta}$. Note that $f$ is an entire function. So by (3.6), (3.7) and (3.3) we find that

$$
\begin{aligned}
T(r, f) & =T(r, G)+S(r, f) \leq 3 T(r, g)+S(r, f) \leq 3 \bar{N}\left(r, \frac{1}{f}\right)+S(r, f) \\
& \leq 3 \bar{N}_{(3}\left(r, \frac{1}{f}\right)+S(r, f) \leq N_{(3}\left(r, \frac{1}{f}\right)+S(r, f) \leq T(r, f)+S(r, f),
\end{aligned}
$$

so that $T(r, f)=3 T(r, g)+S(r, f)$. It follows that every $S(r, f)$ is also an $S(r, g)$ and vice versa. From now on we will write $S(r)$ for the common error term. have

Since any zero of $G=\frac{W \beta^{3}}{f}$ can only occur at a zero of $W$ or a zero of $\beta$, we

$$
\begin{aligned}
\bar{N}\left(r, \frac{1}{G}\right) & \leq \bar{N}\left(r, \frac{1}{W}\right)+\bar{N}\left(r, \frac{1}{\beta}\right) \\
& \leq T(r, W)+T(r, \beta)+O(1)=S(r) .
\end{aligned}
$$

By Lemma 2.1, only three cases are possible. 
CASE 1. $G$ can be expressed as

$$
G=\left(g-\frac{2 \lambda+1}{3}\right)^{3}=g^{3}-(2 \lambda+1) g^{2}+3\left(\frac{2 \lambda+1}{3}\right)^{2} g-\left(\frac{2 \lambda+1}{3}\right)^{3}
$$

From this and (3.7), equating the coefficients of $g$ and $g^{0}$ terms, we obtain

$$
3\left(\frac{2 \lambda+1}{3}\right)^{2} \equiv \lambda(\lambda+2) \text { and } W \beta^{2}-\lambda^{2} \equiv-\left(\frac{2 \lambda+1}{3}\right)^{3}
$$

or, equivalently,

$$
(\lambda-1)^{2} \equiv 0 \text { and } W \beta^{2}-\lambda^{2} \equiv-\left(\frac{2 \lambda+1}{3}\right)^{3} .
$$

That is $W \beta^{2} \equiv 0$. Since $\beta \not \equiv 0$, therefore $W \equiv 0$. Hence $f \equiv f^{\prime}$, a contradiction.

CASE 2. There exists a nonzero small function $\alpha_{0}$ and an integer $\mu$ such that $2 \mu=3$ and

$$
G=\left(g^{2}-2\left(\frac{2 \lambda+1}{3}\right) g+\left(\frac{2 \lambda+1}{3}\right)^{2}+\alpha_{0}\right)^{\mu} .
$$

In this case it is impossible to find an integer number $\mu$ such that $2 \mu=3$.

CASE 3. There exists a nonzero small function $\alpha_{0}$, positive integers $\mu_{1}$ and $\mu_{2}$, and distinct complex numbers $\kappa_{1}$ and $\kappa_{2}$ such that $\mu_{1}+\mu_{2}=3$, $\mu_{1} \kappa_{1}+\mu_{2} \kappa_{2}=0$ and

$$
G=\left(g-\frac{1}{3}(2 \lambda+1)-\kappa_{1} \alpha_{0}\right)^{\mu_{1}}\left(g-\frac{1}{3}(2 \lambda+1)-\kappa_{2} \alpha_{0}\right)^{\mu_{2}} .
$$

Without loss of generality we suppose $\mu_{1}=1$ and $\mu_{2}=2$. Thus $\kappa_{1}+2 \kappa_{2}=0$ and (3.9) becomes

$$
G=\left(g-v_{1}\right)\left(g-v_{2}\right)^{2}
$$

where

$$
v_{1}=\frac{1}{3}(2 \lambda+1)+\kappa_{1} \alpha_{0} \quad \text { and } \quad v_{2}=\frac{1}{3}(2 \lambda+1)-\frac{1}{2} \kappa_{1} \alpha_{0} .
$$

Since $\kappa_{1} \neq 0$ and $\alpha_{0} \not \equiv 0$, therefore $v_{1} \not \equiv v_{2}$. If $v_{1} \equiv 0$, then $v_{2}=\frac{1}{2}(2 \lambda+1)$. From this, (3.10) and (3.7), equating the coefficient of $g$ and $g^{0}$ terms, we get

$$
\lambda \equiv \frac{1}{4} \quad \text { and } \quad W \beta^{2} \equiv \frac{1}{16} .
$$


Then (3.10) reads

$$
\frac{\beta}{16 f}=g\left(g-\frac{3}{4}\right)^{2}
$$

Differentiating (3.11) and then using $\lambda \equiv \frac{1}{4}$, we find that

$$
\frac{\beta}{16 f}\left(g-\frac{1}{4}\right)=-3 g^{\prime}\left(g-\frac{3}{4}\right)\left(g-\frac{1}{4}\right)
$$

and eliminating $\frac{\beta}{16 f}$ between this and (3.11) leads to

$$
g\left(g-\frac{3}{4}\right)^{2}\left(g-\frac{1}{4}\right)=-3 g^{\prime}\left(g-\frac{3}{4}\right)\left(g-\frac{1}{4}\right) .
$$

If $\left(g-\frac{3}{4}\right)\left(g-\frac{1}{4}\right) \equiv 0$, then it is easy to see that $g$ is a constant. From this and (3.11), it follows that $T(r, f)=S(r, f)$, a contradiction. Therefore $\left(g-\frac{3}{4}\right)\left(g-\frac{1}{4}\right)$ $\not \equiv 0$ and (3.12) becomes $g^{\prime}=-\frac{1}{3} g\left(g-\frac{3}{4}\right)$. We can write this as $\frac{g^{\prime}}{g}-\frac{g^{\prime}}{g-\frac{3}{4}}=\frac{1}{4}$ and integrating both sides, we have $g(z)=\frac{3 / 4}{1-c e^{-(1 / 4) z}}$. Integrating again, we
obtain

$$
f(z)=b\left(e^{(1 / 4) z}-c\right)^{3}
$$

where $b$ and $c$ are nonzero constants. But, since $\frac{1}{4}=\lambda=\frac{\beta^{\prime}}{\beta}$, so $\beta(z)=d e^{(1 / 4) z}$, where $d$ is a nonzero constant. Substituting this into (3.13), we conclude that $T(r, f)=S(r, f)$. Again this is a contradiction.

If $v_{2} \equiv 0$, then similarly as the above discussion, we will arrive at the same contradiction. In the following we assume $v_{1} \not \equiv 0$ and $v_{2} \not \equiv 0$. By Lemma 2.2, (3.10) and (3.8) we see that

$$
\begin{aligned}
T(r, g) & \leq \bar{N}\left(r, \frac{1}{g}\right)+\bar{N}\left(r, \frac{1}{g-v_{1}}\right)+\bar{N}\left(r, \frac{1}{g-v_{2}}\right)+S(r, g) \\
& \leq \bar{N}\left(r, \frac{1}{g}\right)+2 \bar{N}\left(r, \frac{1}{G}\right)+S(r) \leq \bar{N}\left(r, \frac{1}{g}\right)+S(r) .
\end{aligned}
$$

We know from $g=\frac{f^{\prime}}{f}$ that the zeros of $g$ can only occur at the zeros of $f^{\prime}$ which are not zero of $f$. Consequently, from (3.3),

$$
\begin{aligned}
T(r, g) & \leq \bar{N}\left(r, \frac{1}{g}\right)+S(r) \leq \bar{N}_{(2}\left(r, \frac{1}{g}\right)+S(r) \\
& \leq \frac{1}{2} N\left(r, \frac{1}{g}\right)+S(r) \leq \frac{1}{2} T(r, g)+S(r)
\end{aligned}
$$

a contradiction, and the proof of Theorem 1.2 is complete. 


\subsection{Proof of Theorem 1.4}

Consider the auxiliary function

$$
h=\frac{\Delta^{2}(f)}{\left(f-\beta_{1}\right)\left(f-\beta_{2}\right)} .
$$

From Lemma 2.3 we know that $\Delta(f) \not \equiv 0$ and

$$
m(r, h) \leq m\left(r, \frac{\Delta(f)}{f-\beta_{1}}\right)+m\left(r, \frac{\Delta(f)}{f-\beta_{2}}\right)=S(r, f) .
$$

From the hypotheses of Theorem 1.4 and Lemma 2.2 we deduce that

$$
\begin{aligned}
T(r, f) & \leq \sum_{j=1}^{2} \bar{N}\left(r, \frac{1}{f-\beta_{j}}\right)+\bar{N}(r, f)+S(r, f) \\
& =\sum_{j=1}^{2} \bar{N}_{(2}\left(r, \frac{1}{f-\beta_{j}}\right)+S(r, f) \\
& \leq \sum_{j=1}^{2} \frac{1}{2} N_{(2}\left(r, \frac{1}{f-\beta_{j}}\right)+S(r, f) \leq T(r, f)+S(r, f) .
\end{aligned}
$$

Therefore

$$
T(r, f)=\sum_{j=1}^{2} \bar{N}_{(2}\left(r, \frac{1}{f-\beta_{j}}\right)+S(r, f)=\frac{1}{2} \sum_{j=1}^{2} N_{(2}\left(r, \frac{1}{f-\beta_{j}}\right)+S(r, f) .
$$

This implies that

$$
T(r, f)=2 \bar{N}_{=2}\left(r, \frac{1}{f-\beta_{1}}\right)+S(r, f)=2 \bar{N}_{=2}\left(r, \frac{1}{f-\beta_{2}}\right)+S(r, f) .
$$

From Lemma 2.3, it is easy to see that

$$
\Delta(f)=\left(\frac{f-\beta_{1}}{\beta_{2}-\beta_{1}}\right)^{\prime}\left(\beta_{2}-\beta_{1}\right)^{2}=\left(\frac{f-\beta_{2}}{\beta_{2}-\beta_{1}}\right)^{\prime}\left(\beta_{2}-\beta_{1}\right)^{2} .
$$

By (3.17) and (3.18), if any zero of $f-\beta_{j}(j=1,2)$ has multiplicity two, then it must be a zero of $\Delta^{2}(f)$ with multiplicity two. Thus, from (3.15) we get $N(r, h)=S(r, f)$. Together with (3.16) we have

$$
T(r, h)=S(r, f) \text {. }
$$

We consider the transformation

$$
F=\frac{f-\beta_{1}}{\beta_{2}-\beta_{1}}
$$


Substitution of expressions (3.20) and (3.18) into (3.15) now tells us that

$$
\frac{h}{\left(\beta_{2}-\beta_{1}\right)^{2}}=\frac{F^{\prime 2}}{F(F-1)}=\left(\frac{F^{\prime}}{F}\right)^{2}\left(\frac{F}{F-1}\right)
$$

or

$$
H=L^{2}+\delta
$$

where $\delta=\frac{-h}{\left(\beta_{2}-\beta_{1}\right)^{2}}, H=\frac{\delta}{F}$ and $L=\frac{F^{\prime}}{F}$. Differentiating (3.21) once we obtain

$$
H^{\prime}=2 L L^{\prime}+\delta^{\prime}
$$

On the other hand differentiating $H=\frac{\delta}{F}$ we find that

$$
H^{\prime}=\frac{\delta}{F}\left(\frac{\delta^{\prime}}{\delta}-L\right)
$$

and eliminating $H^{\prime}$ between (3.22) and (3.23) leads to

$$
2 L L^{\prime}=-L^{3}+\frac{\delta^{\prime}}{\delta} L^{2}-\delta L .
$$

If $L \equiv 0$, then $F$ is a constant and so $T(r, f)=S(r, f)$ a contradiction. Therefore $L \not \equiv 0$, and (3.24) becomes

$$
2 L^{\prime}=-L^{2}+\frac{\delta^{\prime}}{\delta} L-\delta
$$

Let $z_{1}$ be a zero of $f-\beta_{1}$ with multiplicity two and $\beta_{i}\left(z_{1}\right) \neq 0, \infty(i=1,2)$, $\left(\beta_{2}-\beta_{1}\right)\left(z_{1}\right) \neq 0, \infty$. Then the Taylor expansion of $F$ about $z_{1}$ is

$$
F(z)=a_{2}\left(z-z_{1}\right)^{2}+a_{3}\left(z-z_{1}\right)^{3}+a_{4}\left(z-z_{1}\right)^{4}+\cdots, \quad a_{2} \neq 0 .
$$

Hence

$$
\begin{gathered}
L=\frac{F^{\prime}}{F}=2\left(z-z_{1}\right)^{-1}+\frac{a_{3}}{a_{2}}+\left[2 \frac{a_{4}}{a_{2}}-\left(\frac{a_{3}}{a_{2}}\right)^{2}\right]\left(z-z_{1}\right)+O\left(\left(z-z_{1}\right)^{2}\right), \\
L^{\prime}=-2\left(z-z_{1}\right)^{-2}+2 \frac{a_{4}}{a_{2}}-\left(\frac{a_{3}}{a_{2}}\right)^{2}+O\left(\left(z-z_{1}\right)\right)
\end{gathered}
$$

and

$$
L^{2}=4\left(z-z_{1}\right)^{-2}+4 \frac{a_{3}}{a_{2}}\left(z-z_{1}\right)^{-1}+8 \frac{a_{4}}{a_{2}}-3\left(\frac{a_{3}}{a_{2}}\right)^{2}+O\left(\left(z-z_{1}\right)\right) .
$$

Substituting (3.26), (3.27) and (3.28) into (3.25) gives

$$
\frac{a_{3}}{a_{2}}=\frac{\delta^{\prime}}{2 \delta} \quad \text { and } \quad \frac{a_{4}}{a_{2}}=\frac{7}{48}\left(\frac{\delta^{\prime}}{\delta}\right)^{2}-\frac{1}{12} \delta
$$


From (3.27) we find that

$$
\frac{L^{\prime \prime}}{L^{\prime}}=-2\left(z-z_{1}\right)^{-1}-\left[2 \frac{a_{4}}{a_{2}}-\left(\frac{a_{3}}{a_{2}}\right)^{2}\right]\left(z-z_{1}\right)+O\left(\left(z-z_{1}\right)^{2}\right) .
$$

Hence

$$
\left(\frac{L^{\prime \prime}}{L^{\prime}}\right)^{\prime}=2\left(z-z_{1}\right)^{-2}-\left[2 \frac{a_{4}}{a_{2}}-\left(\frac{a_{3}}{a_{2}}\right)^{2}\right]+O\left(\left(z-z_{1}\right)\right)
$$

and

$$
\left(\frac{L^{\prime \prime}}{L^{\prime}}\right)^{2}=4\left(z-z_{1}\right)^{-2}+4\left[2 \frac{a_{4}}{a_{2}}-\left(\frac{a_{3}}{a_{2}}\right)^{2}\right]+O\left(\left(z-z_{1}\right)\right) .
$$

We set

$$
\eta=\left(\frac{L^{\prime \prime}}{L^{\prime}}\right)^{2}-2\left(\frac{L^{\prime \prime}}{L^{\prime}}\right)^{\prime}
$$

From (3.25) it is easy to see that $N\left(r, L^{\prime}\right)=2 N(r, L)+S(r, f)$. This implies that $N(r, L)=\bar{N}(r, L)+S(r, f)$. From this we conclude that

$$
N_{(2}(r, L)=S(r, f) \text {. }
$$

Substituting (3.30) and (3.31) into (3.32) and then using (3.29) we arrive at

If $\eta \not \equiv \frac{1}{4}\left(\frac{\delta^{\prime}}{\delta}\right)^{2}-\delta$, then

$$
\eta=\frac{1}{4}\left(\frac{\delta^{\prime}}{\delta}\right)^{2}-\delta+O\left(\left(z-z_{1}\right)\right)
$$

$$
\begin{aligned}
\bar{N}_{=2}\left(r, \frac{1}{f-\beta_{1}}\right) & \leq N\left(r, \frac{1}{\eta-\frac{1}{4}\left(\frac{\delta^{\prime}}{\delta}\right)^{2}+\delta}\right)+S(r, f) . \\
& \leq T\left(r, \eta-\frac{1}{4}\left(\frac{\delta^{\prime}}{\delta}\right)^{2}+\delta\right)+S(r, f) \leq T(r, \eta)+S(r, f) .
\end{aligned}
$$

Combining this with (3.33) and Lemma 2.4 yields

$$
\bar{N}_{=2}\left(r, \frac{1}{f-\beta_{1}}\right) \leq 2 \bar{N}\left(r, \frac{1}{L^{\prime}}\right)+S(r, f) .
$$
Together with $(3.17)$ we have $T(r, f) \leq 4 \bar{N}\left(r, \frac{1}{L^{\prime}}\right)+S(r, f)$. This is (1.4). In
the following, we assume that

$$
\eta \equiv \frac{1}{4}\left(\frac{\delta^{\prime}}{\delta}\right)^{2}-\delta
$$


On the other hand, differentiating (3.25) twice we obtain

$$
2 L^{\prime \prime}=L\left[-2 L^{\prime}+\left(\frac{\delta^{\prime}}{\delta}\right)^{\prime}\right]+\frac{\delta^{\prime}}{\delta} L^{\prime}-\delta^{\prime}
$$

and

$$
2 L^{\prime \prime \prime}=-2 L^{\prime 2}-L\left[2 L^{\prime \prime}-\left(\frac{\delta^{\prime}}{\delta}\right)^{\prime \prime}\right]+2\left(\frac{\delta^{\prime}}{\delta}\right)^{\prime} L^{\prime}+\frac{\delta^{\prime}}{\delta} L^{\prime \prime}-\delta^{\prime \prime} .
$$

Suppose that $z_{2}$ is a zero of $f-\beta_{2}$ with multiplicity two and $\beta_{i}\left(z_{2}\right) \neq 0, \infty$ $(i=1,2),\left(\beta_{2}-\beta_{1}\right)\left(z_{2}\right) \neq 0, \infty$. Then $F(z)=1+O\left(\left(z-z_{2}\right)^{2}\right)$ and so $L\left(z_{2}\right)=0$. Thus we deduce from (3.25), (3.35), (3.36) and (3.32) that

$$
\eta=3\left(\frac{L^{\prime \prime}}{L^{\prime}}\right)^{2}-2 \frac{L^{\prime \prime \prime}}{L^{\prime}}=\frac{29}{4}\left(\frac{\delta^{\prime}}{\delta}\right)^{2}-4 \frac{\delta^{\prime \prime}}{\delta}-\delta+O\left(\left(z-z_{2}\right)\right)
$$

As in the above discussion, we find that either (1.4) holds or

$$
\eta \equiv \frac{29}{4}\left(\frac{\delta^{\prime}}{\delta}\right)^{2}-4 \frac{\delta^{\prime \prime}}{\delta}-\delta
$$

If we now eliminate $\eta$ between (3.37) and (3.34) we arrive at

$$
7 \frac{\delta^{\prime 2}}{\delta}=4 \delta^{\prime \prime}
$$

If $\delta^{\prime} \not \equiv 0$, then from (3.38) we have $7 \frac{\delta^{\prime}}{\delta}=4 \frac{\delta^{\prime \prime}}{\delta^{\prime}}$. By integrating once,

$$
\delta^{7}=c \delta^{\prime 4}
$$

where $c$ is a nonzero constant. From this we conclude that

$$
3 m(r, \delta)=m\left(r, c\left(\frac{\delta^{\prime}}{\delta}\right)^{4}\right)=4 m\left(r, \frac{\delta^{\prime}}{\delta}\right)+O(1)=4 S(r, \delta)+O(1)=S(r, \delta) .
$$

It follows from (3.39) that if $z_{\infty}$ is a pole of $\delta$ with multiplicity $p(\geq 1)$, then $O\left(\left(z-z_{\infty}\right)^{-7 p}\right)=O\left(\left(z-z_{\infty}\right)^{-4(p+1)}\right)$. Hence $p=\frac{4}{3}$, which contradicts with $p$ being an integer. Therefore $N(r, \delta) \equiv 0$. Together with (3.40) we get $T(r, \delta)=$ $S(r, \delta)$ and this gives us $\delta$ is a nonzero constant. That is $\delta^{\prime} \equiv 0$. This and (3.25) imply that $\frac{L^{\prime}}{L-c}-\frac{L^{\prime}}{L+c}=-c$, where $c=i \sqrt{\delta}$. By integration, we obtain $L=\frac{-c e^{c z}}{B-e^{c z}}+\frac{c B e^{-c z}}{1-B e^{-c z}}$. Since $L=\frac{F^{\prime}}{F}$, it follows from the integration that $F(z)=A e^{-c z}\left(1-B e^{c z}\right)^{2}$, where $A$ and $B$ are nonzero constants. From this and (3.20) we have

$$
f(z)-\beta_{1}(z)=A\left(\beta_{2}(z)-\beta_{1}(z)\right) e^{-c z}\left(1-B e^{c z}\right)^{2} .
$$


We rewrite this in the form

$$
f(z)-\beta_{2}(z)=A\left(\beta_{2}(z)-\beta_{1}(z)\right) e^{-c z}\left[1-\left(2 B+\frac{1}{A}\right) e^{c z}+B^{2} e^{2 c z}\right]
$$

which, in view of (3.17), leads to $A=\frac{-1}{4 B}$. Substituting this into (3.41) and (3.42) we arrive at (1.2) and (1.3) respectively. This completes the proof of Theorem 1.4.

Acknowledgement. The author wishes to thank the referee for careful reading of this paper which led to useful comments.

\section{REFERENCES}

[1] A. H. H. Al-Khaladi, Meromorphic functions and their kth derivative share only one small function CM, Novi Sad J. Math. 44 (2014), 21-35.

[2] W. K. Hayman, Meromorphic functions, Clarendon Press, Oxford, 1964, 47-48.

[3] E. Mues and N. Steinmetz, Meromorphe Funktionen die mit ihrer Ableitung Werte teilen, Manuscripta Math. 29 (1979), 195-206.

[4] E. Mues and N. Steinmetz, The theorem of Tumura-Clunie for meromorphic functions, J. London Math. Soc. 23 (1981), 113-122.

[5] G. QIU, Uniqueness of entire functions that share some small functions, Kodai Math. J. 23 (2000), $1-11$.

[6] L. A. Rubel and C. C. YAnG, Values shared by an entire function and its derivative, Lecture notes in mathematics 599, Springer-Verlag, New York, 1977, 101-103.

[ 7 ] A. SCHweizer, Entire functions sharing simple $a$-points with their first derivative, Houston J. Math. 39 (2013), 1137-1148.

[ 8 ] C. C. YANG AND H. X. YI, Uniqueness theory of meromorphic functions, Kluwer Academic Publishers, 2004.

[9] J. H. Zheng AND S. P. WANG, Unicity of meromorphic functions and their derivatives, Adv. in Math. 21 (1992), 334-341.

Amer H. H. Al-Khaladi

Department of Mathematics

College of Basic Education

Tikrit University

P. O. Box 42, Tikrit

IRAQ

E-mail: ameralkhaladi@yahoo.com 